\title{
The role of lung ultrasound in the diagnosis of pneumonia in acutely ill patients
}

\author{
M Biancardi ${ }^{*}$, B D $^{\prime}$ Agostino ${ }^{1}$, C Bonfardeci ${ }^{2}$ \\ From 7th WINFOCUS Italian Congress on Ultrasound in Emergency, Anaesthesiology and Critical Care \\ Lodi, Italy. 26-29 March 2014
}

\section{Background}

The differential diagnosis of patients in the emergency department who show symptoms of sepsis and suspected pneumonia may be difficult because of the low sensitivity and specificity of supine chest $\mathrm{X}$ rays (SCXR) and physical examination.

\section{Objective}

A retrospective assessment of the usefulness of lung ultrasound (LU) in the diagnosis of pneumonia in patients with signs and symptoms of sepsis and respiratory disease.

\section{Patients and methods}

2 groups of patients were considered:

Group A: 22 patients with a positive first SCXR (SCXR1) for pneumonia

Group B: 18 patients with a negative SCXR1 for pneumonia, but who were subsequently diagnosed with pneumonia during their hospital stay following a second SCXR (SCXR2) or CT.

Lung ultrasound examination was performed within 24 hours of admission, searching for direct and indirect echographical signs of pneumonia: focal B lines (FBL), pleural effusion (PE) and images of lung consolidation (LC).

\section{Results}

See table 1 .

\section{Conclusions}

In our observational study, LU showed high sensitivity in the diagnosis of pneumonia:

95\% in patients already diagnosed using SCXR, and $100 \%$ of early diagnosis of pneumonia in patients whose diagnosis was subsequently confirmed during hospitalization.
Table 1

\begin{tabular}{lll}
\hline & Group A (\%) & Group B (\%) \\
\hline Male/Female & $11 / 11$ & $7 / 11$ \\
\hline MEAN AGE & 74,8 & 76,8 \\
\hline SCXR1 pos & 22 & 0 \\
\hline SCXR2 or CT pos & - & 18 \\
\hline FBL pos & $9(41)$ & $10(50)$ \\
\hline FBL neg & $13(59)$ & $10(50)$ \\
\hline PE pos & $17(78)$ & $16(80)$ \\
\hline PE neg & $5(22)$ & $4(20)$ \\
\hline LC pos & $19(87)$ & $20(100)$ \\
\hline LC neg & $3(13)$ & $0(0)$ \\
\hline LC pos or FBL pos & $21(95)$ & - \\
\hline LU impact on therapy & No & $20(100)$ \\
\hline
\end{tabular}

\section{Authors' details}

'Medicina 1 dept, Ospedale San Carlo Borromeo, Milano, Italy. ${ }^{2}$ Medicina d' Urgenza, Ospedale San Carlo Borromeo, Milano, Italy.

Published: 27 August 2014

\section{References}

1. Lichtenstein DA: Relevance of Lung Ultrasound in the Diagnosis of Acute Respiratory Failure - The Blue Protocol. Chest 2008, 134:117-125.

2. Essayag $Y$, et al: Diagnostic value of chest radiographs in bedridden patients suspected of having pneumonia. Am J Med 2010, 123:88. e 1-5.

3. Reissig A: Sonographic Diagnosis and Follow-up of Pneumoniai: A Prospective Study. Respiration 2007, 74:537-547.

4. Mathis G: Thoraxsonography - Part II: Peripheral Pulmonary Consolidation. Ultrasound in Med. \& Biol 1997, 23(8):1141-1153.

5. Volpicelli G: Detection of sonographic B lines in patients with normal lungs or radiographic alveolar consolidation. Med Sci Monitor 2008, 14(3): CR 122-128.

doi:10.1186/2036-7902-6-S2-A2

Cite this article as: Biancardi et al:: The role of lung ultrasound in the diagnosis of pneumonia in acutely ill patients. Critical Ultrasound Journal 2014 6(Suppl 2):A2.

* Correspondence: marco.biancardi@katamail.com

${ }^{1}$ Medicina 1 dept, Ospedale San Carlo Borromeo, Milano, Italy

Full list of author information is available at the end of the article 